\title{
FINDING THE LIMIT OF INCOMPLETENESS I
}

\author{
YONG CHENG
}

\begin{abstract}
In this paper, we examine the limit of applicability of Gödel's first incompleteness theorem (G1 for short). We first define the notion "G1 holds for the theory T". This paper is motivated by the following question: can we find a theory with a minimal degree of interpretation for which G1 holds. To approach this question, we first examine the following question: is there a theory $T$ such that Robinson's $\mathbf{R}$ interprets $T$ but $T$ does not interpret $\mathbf{R}$ (i.e. $T$ is weaker than $\mathbf{R}$ w.r.t. interpretation) and G1 holds for $T$ ? In this paper, we show that there are many such theories based on Jeřábek's work using some model theory. We prove that for each recursively inseparable pair $\langle A, B\rangle$, we can construct a r.e. theory $U_{\langle A, B\rangle}$ such that $U_{\langle A, B\rangle}$ is weaker than $\mathbf{R}$ w.r.t. interpretation and $\mathrm{G} 1$ holds for $U_{\langle A, B\rangle}$. As a corollary, we answer a question from Albert Visser. Moreover, we prove that for any Turing degree $\mathbf{0}<\mathbf{d}<\mathbf{0}^{\prime}$, there is a theory $T$ with Turing degree $\mathbf{d}$ such that G1 holds for $T$ and $T$ is weaker than $\mathbf{R}$ w.r.t. Turing reducibility. As a corollary, based on Shoenfield's work using some recursion theory, we show that there is no theory with a minimal degree of Turing reducibility for which G1 holds.
\end{abstract}

\section{INTRODUCTION}

Gödel's incompleteness theorem is one of the most remarkable results in the foundation of mathematics and has had great influence in logic, philosophy, mathematics, physics and computer science, as discussed in [25], 31. Gödel proved his incompleteness theorems in [12 for a certain formal system $\mathbf{P}$ related to Russell-Whitehead's Principia Mathematica and based on the simple theory of types over the natural number series and the Dedekind-Peano axioms (see [1, p.3). The following theorem is a modern reformulation of Gödel's first incompleteness theorem (where PA refers to the first order theory commonly known as Peano Arithmetic).

Theorem 1.1 (Gödel-Rosser, First incompleteness theorem (G1)). If $T$ is a recursively axiomatized consistent extension of $\mathbf{P A}$, then $T$ is incomplete.

2010 Mathematics Subject Classification. 03F40, 03F30, 03D35.

Key words and phrases. Gödel's first incompleteness theorem, Interpretation, Essential undecidability, Robinson's R.

I would like to thank Prof. Albert Visser for inspiring discussions of the topic in this paper, insightful comments on the original version of the paper and for introducing some papers to me. I would also like to thank Prof. Emil Jeřábek for his patient explanations of his work to me. I would like to thank Prof. Ali Enayat and Prof. V.Yu. Shavrukov for their comments on my paper. I would like to thank the referee for providing detailed and helpful comments for improvements. This paper is the research result of The National Social Science Fund of China for general project "Research on the limit of incompleteness and the intensional problem of incompleteness" (project no: 18BZX131). I would like to thank the fund support by The National Social Science Fund of China for general project. 
The following is a well known open question about G1.

Question 1.2. Exactly how much arithmetical information from PA is needed for the proof of $\mathrm{G} 1$ ?

The notion of interpretation provides us a method to compare different theories in different languages (for the definition of interpretation, see Section 21). Given theories $S$ and $T, S \unlhd T$ denotes that $S$ is interpretable in $T$ (or $T$ interprets $S$ ) and $S \triangleleft T$ denotes that $S$ is interpretable in $T$ but $T$ is not interpretable in $S$. We say that the theory $S$ and $T$ are mutually interpretable if $S \unlhd T$ and $T \unlhd S$. In this paper, we equate a set of sentences $\Gamma$ in the language of arithmetic with the set of Gödel's numbers of sentences in $\Gamma$ (see Section 2 for more details about Gödel's number). Given two arithmetic theories $U$ and $V, U \leq_{T} V$ denotes that the theory $U$ as a set of natural numbers is Turing reducible to the theory $V$ as a set of natural numbers, and $U<_{T} V$ denotes that $U \leq_{T} V$ but $V \leq_{T} U$.

Note that G1 can be generalized via interpretability: there exists a weak recursively axiomatizable consistent subtheory $T$ (e.g. Robinson Arithmetic Q) of PA such that for each recursively axiomatizable consistent theory $S$, if $T$ is interpretable in $S$, then $S$ is incomplete (see [35]). To generalize this fact, in the following, we propose a general new notion "G1 holds for the theory T".

\section{Definition 1.3.}

(1) Let $T$ be a recursively axiomatizable consistent theory. We say that G1 holds for $T$ if for any recursively axiomatizable consistent theory $S$, if $T$ is interpretable in $S$, then $S$ is incomplete.

(2) We say the theory $S$ has a minimal degree of interpretation if there is no theory $T$ such that $T \triangleleft S$.

(3) We say the theory $S$ has a minimal degree of Turing reducibility if there is no theory $V$ such that $V<_{T} S$.

(4) In this paper, whenever we say that the theory $S$ is weaker than the theory $T$ w.r.t. interpretation, this means that $S \triangleleft T$.

(5) In this paper, whenever we say that the theory $S$ is weaker than the theory $V$ w.r.t. Turing reducibility, this means that $S<_{T} V$.

Toward Question 1.2, in this project, we want to examine the following question:

Question 1.4. Can we find a theory $S$ such that G1 holds for $S$ and $S$ has a minimal degree of interpretation?

It is well known that G1 holds for Robinson Arithmetic Q (see [35]). From [35], G1 also holds for Robinson's theory $\mathbf{R}$ (for definitions of $\mathbf{Q}$ and $\mathbf{R}$, we refer to Section 21). In Section 2, we review some theories mutually interpretable with $\mathbf{Q}$ for which $\mathrm{G} 1$ holds, and some theories mutually interpretable with $\mathbf{R}$ for which G1 holds. As the first step toward Question 1.4, we propose the following question:

Question 1.5. Can we find a theory $S$ such that G1 holds for $S$ and $S \triangleleft \mathbf{R}$ ?

We find that Jeřábek essentially answered this question in [21]. In this paper, we show that there are many examples of such a theory $S$ : for each 
recursively inseparable pair $\langle A, B\rangle$, we can construct a r.e. theory $U_{\langle A, B\rangle}$ such that G1 holds for $U_{\langle A, B\rangle}$ and $U_{\langle A, B\rangle} \triangleleft \mathbf{R}$ based on Jeřábek's work using some model theory. As a corollary, we answer a question from Albert Visser. For Question 1.4, if we consider the degree of Turing reducibility instead of the degree of interpretation, the answer becomes easier. We show that for any Turing degree $0<\mathbf{d}<0^{\prime}$, there is a theory $S$ such that G1 holds for $S$, $S<_{T} \mathbf{R}$ and $S$ has Turing degree $\mathbf{d}$ based on Shoenfield's work using some recursion theory. As a corollary, there is no theory with a minimal degree of Turing reducibility for which G1 holds.

The structure of this paper is as follows. In Section 1, we introduce our research questions and main results of this paper. In Section 2, we list some basic notions and facts we use in this paper and give a review of theories weaker than PA w.r.t. interpretation in the literature for which G1 holds. In Section 3, we examine the limit of applicability of G1 w.r.t. interpretation. We prove Theorem 3.12 and answer a question from Albert Visser. In Section 4, we examine the limit of applicability of G1 w.r.t. Turing reducibility and prove Theorem 4.5 and Corollary 4.6.

\section{Preliminaries}

In this section, we review some basic notions and facts used in this paper. Our notations are standard. For books on Gödel's incompleteness theorem, we refer to [8], 25], [24], 31], 22] and [4]. For survey papers on Gödel's incompleteness theorem, we refer to [1], [23], [32], [41], [5] and [6]. For meta-mathematics of subsystems of PA, we refer to [18].

In this paper, a language consists of an arbitrary number of relation and function symbols of arbitrary finite arity 1$]$ For a given theory $T$, we use $L(T)$ to denote the language of $T$ and often equate $L(T)$ with the list of non-logical symbols of the language. For a formula $\phi$ in $L(T)$, let $T \vdash \phi$ denote that $\phi$ is provable in $T$ (i.e., there is a finite sequence of formulas $\left\langle\phi_{0}, \cdots, \phi_{n}\right\rangle$ such that $\phi_{n}=\phi$, and for any $0 \leq i \leq n$, either $\phi_{i}$ is an axiom of $T$ or $\phi_{i}$ follows from some $\phi_{j}(j<i)$ by using one inference rule). Theory $T$ is consistent if no contradiction is provable in $T$. A formula $\phi$ is independent of $T$ if $T \nvdash \phi$ and $T \nvdash \neg \phi$. A theory $T$ is incomplete if there is a sentence $\phi$ in $L(T)$ such that $\phi$ is independent of $T$; otherwise, $T$ is complete (i.e., for any sentence $\phi$ in $L(T)$, either $T \vdash \phi$ or $T \vdash \neg \phi$ ).

In this paper, we understand that each theory $T$ comes with a fixed arithmetization. Let $T$ be a recursively axiomatizable theory. Under this fixed arithmetization, we could establish the one-to-one correspondence between formulas of $L(T)$ and natural numbers. Under this correspondence, we can translate metamathematical statements about the formal theory $T$ into statements about natural numbers. Furthermore, fundamental metamathematical relations can be translated in this way into certain recursive relations, hence into relations representable in the theory $T$. Consequently, one can speak about a formal system of arithmetic and about its properties as a theory in the system itself! This is the essence of Gödel's idea of arithmetization. Under arithmetization, any formula or finite sequence

\footnotetext{
${ }^{1}$ We may view nullary functions as constants and nullary relations as propositional variables.
} 
of formulas can be coded by a natural number (this code is called a Gödel number). In this paper, we use $\ulcorner\phi\urcorner$ to denote the Gödel number of $\phi$. For details of arithmetization, we refer to [25].

Given a set of sentences $\Sigma$, we say $\Sigma$ is recursive if the set of Gödel numbers of sentences in $\Sigma$ is recursive. A theory $T$ is decidable if the set of sentences provable in $T$ is recursive; otherwise it is undecidable. A theory $T$ is recursively axiomatizable if it has a recursive set of axioms (i.e. the set of Gödel numbers of axioms of $T$ is recursive) and it is finitely axiomatized if it has a finite set of axioms. A theory $T$ is recursively enumerable (r.e.) if it has a recursively enumerable set of axioms. A theory $T$ is essentially undecidable if any recursively axiomatizable consistent extension of $T$ in the same language is undecidable. A theory $T$ is essentially incomplete if any recursively axiomatizable consistent extension of $T$ in the same language is incomplete. The theory of completeness/incompleteness is closely related to the theory of decidability/undecidability. A theory $T$ is minimal essentially undecidable if $T$ is essentially undecidable and if deleting any axiom of $T$, the remaining theory is no longer essentially undecidable. A theory $T$ is locally finitely satisfiable if every finitely axiomatized subtheory of $T$ has a finite model.

A $n$-ary relation $R\left(x_{1}, \cdots, x_{n}\right)$ on $\mathbb{N}^{n}$ is representable in $T$ iff there is a formula $\phi\left(x_{1}, \cdots, x_{n}\right)$ such that $T \vdash \phi\left(\overline{m_{1}}, \cdots, \overline{m_{n}}\right)$ if $R\left(m_{1}, \cdots, m_{n}\right)$ holds (for $n \in \mathbb{N}$, we denote by $\bar{n}$ the corresponding numeral for $n$ in $L(\mathbf{P A})$ ) and $T \vdash \neg \phi\left(\overline{m_{1}}, \cdots, \overline{m_{n}}\right)$ if $R\left(m_{1}, \cdots, m_{n}\right)$ does not hold. We say that a partial function $f\left(x_{1}, \cdots, x_{n}\right)$ on $\mathbb{N}^{n}$ is representable in $T$ iff there is a formula $\varphi\left(x_{1}, \cdots, x_{n}, y\right)$ such that $T \vdash \forall y\left(\varphi\left(\overline{a_{1}}, \cdots, \overline{a_{n}}, y\right) \leftrightarrow y=\bar{m}\right)$ whenever $a_{1}, \cdots, a_{n}, m \in \mathbb{N}$ are such that $f\left(a_{1}, \cdots, a_{n}\right)=m$.

For the definitions of translation and interpretation, we follow [21]. Let $T$ be a theory in a language $L(T)$, and $S$ a theory in a language $L(S)$. In its most simple form, a translation $I$ of language $L(T)$ into language $L(S)$ is specified by:

(1) an $L(S)$-formula $\delta_{I}(x)$ denoting the domain of $I$;

(2) for each relation symbol $R$ of $L(T)$, an $L(S)$-formula $R_{I}$ of the same arity;

(3) for each function symbol $F$ of $L(T)$ of arity $k$, an $L(S)$-formula $F_{I}$ of arity $k+1$.

If $\phi$ is an $L(T)$-formula, its $I$-translation $\phi^{I}$ is an $L(S)$-formula constructed as follows: we reformulate the formula in an equivalent way so that function symbols only occur in atomic subformulas of the form $F(\bar{x})=y$ where $x_{i}, y$ are variables; then we replace each such atomic formula with $F_{I}(\bar{x}, y)$, we replace each atomic formula of the form $R(\bar{x})$ with $R_{I}(\bar{x})$, and we restrict all quantifiers and free variables to objects satisfying $\delta_{I}$. Moreover, we rename bound variables to avoid variable clashes during the process (see [21]).

A translation $I$ of $L(T)$ into $L(S)$ is an interpretation of $T$ in $S$ if $S$ proves:

(1) for each function symbol $F$ of $L(T)$ of arity $k$, the formula expressing that $F_{I}$ is total on $\delta_{I}: \forall x_{0}, \cdots \forall x_{k-1}\left(\delta_{I}\left(x_{0}\right) \wedge \cdots \wedge \delta_{I}\left(x_{k-1}\right) \rightarrow \exists y\left(\delta_{I}(y) \wedge\right.\right.$ $\left.\left.F_{I}\left(x_{0}, \cdots, x_{k-1}, y\right)\right)\right)$; 
(2) the $I$-translations of all theorems of $T$, and axioms of equality.

The simplified picture of translations and interpretations above actually describes only one-dimensional, parameter-free, and one-piece translations (see [21]). For the precise and technical definitions of a multi-dimensional interpretation, an interpretation with parameters and a piece-wise interpretation, we refer to [37, [39] and [40] for the details.

A theory $T$ is interpretable in a theory $S$ if there exists an interpretation of $T$ in $S$. If $T$ is interpretable in $S$, then all sentences provable (refutable) in $T$ are mapped, by the interpretation function, to sentences provable (refutable) in $S$. Interpretability can be accepted as a measure of strength of different theories. If $S \triangleleft T$, then $S$ can be considered weaker than $T$ w.r.t. interpretation; if $S$ and $T$ are mutually interpretable, then $T$ and $S$ are equally strong w.r.t. interpretation. The theory $U$ weakly interprets the theory $V$ (or $V$ is weakly interpretable in $U$ ) if $V$ is interpretable in some consistent extension of $U$ in the same language (or equivalently, for some interpretation $\tau$, the theory $U+V^{\tau}$ is consistent).

A general method for establishing the undecidability of theories is developed in [35]. The following theorem provides us two methods to prove the essentially undecidability of a theory via interpretation and representability.

\section{Theorem 2.1.}

(1) ([35, Theorem 7, p.22]) Let $T_{1}$ and $T_{2}$ be two theories such that $T_{1}$ is consistent and $T_{2}$ is interpretable in $T_{1}$. We then have: if $T_{2}$ is essentially undecidable, then $T_{1}$ is also essentially undecidable.

(2) (35, Corollary 2, p.49]) If $T$ is a consistent theory in which all recursive functions are representable, then $T$ is essentially undecidable.

In Section 3, we will show that G1 holds for the theory $T$ iff $T$ is essentially undecidable. In the following, we review some theories from the literature which are weaker than PA w.r.t. interpretation and which are essentially undecidable (i.e. G1 holds for them).

Robinson Arithmetic $\mathbf{Q}$ was introduced in 35 by Tarski, Mostowski and Robinson as a base axiomatic theory for investigating incompleteness and undecidability.

Definition 2.2. Robinson Arithmetic $\mathbf{Q}$ is defined in the language $\{\mathbf{0}, \mathbf{S},+, \cdot\}$ with the following axioms:

$$
\begin{aligned}
& \mathbf{Q}_{1}: \forall x \forall y(\mathbf{S} x=\mathbf{S} y \rightarrow x=y) ; \\
& \mathbf{Q}_{2}: \forall x(\mathbf{S} x \neq \mathbf{0}) ; \\
& \mathbf{Q}_{3}: \forall x(x \neq \mathbf{0} \rightarrow \exists y x=\mathbf{S} y) ; \\
& \mathbf{Q}_{4}: \forall x(x+\mathbf{0}=x) ; \\
& \mathbf{Q}_{5}: \forall x \forall y(x+\mathbf{S} y=\mathbf{S}(x+y)) ; \\
& \mathbf{Q}_{6}: \forall x(x \cdot \mathbf{0}=\mathbf{0}) ; \\
& \mathbf{Q}_{7}: \forall x \forall y(x \cdot \mathbf{S} y=x \cdot y+x) .
\end{aligned}
$$

Robinson Arithmetic $\mathbf{Q}$ is very weak and inadequate to formalize arithmetic: for instance, $\mathbf{Q}$ does not even prove that addition is associative. Robinson showed that any consistent theory that interprets $\mathbf{Q}$ is undecidable and hence $\mathbf{Q}$ is essentially undecidable (see [35]). The fact that $\mathbf{Q}$ is essentially undecidable is very useful and can be used to prove the essentially 
undecidability of other theories via Theorem 2.1 . Since $\mathbf{Q}$ is finitely axiomatized, it follows that any theory that weakly interprets $\mathbf{Q}$ is undecidable. In fact, $\mathbf{Q}$ is minimal essentially undecidable in the sense that if deleting any axiom of $\mathbf{Q}$, then the remaining theory is not essentially undecidable and has a complete decidable extension (see [35, Theorem 11, p.62]). Nelson [26] embarked on a program of investigating how much mathematics can be interpreted in Robinson Arithmetic $\mathbf{Q}$ : what can be interpreted in $\mathbf{Q}$ but also what cannot be interpreted in $\mathbf{Q}$. In fact, $\mathbf{Q}$ represents a rich degree of interpretability since a lot of stronger theories are interpretable in it as it can be shown (e.g. using Solovay's method of shortening cuts (see [15]), one can show that $\mathbf{Q}$ interprets fairly strong theories like $I \Delta_{0}+\Omega_{1}$ on a definable cut). The Lindenbaum algebras of all recursively enumerable theories that interpret $\mathbf{Q}$ are recursively isomorphic (see Pour-El and Kripke [27]).

The theory $\mathbf{P A}$ consists of axioms $\mathbf{Q}_{1}-\mathbf{Q}_{2}, \mathbf{Q}_{4}-\mathbf{Q}_{7}$ in Definition 2.2 and the following axiom scheme of induction: $(\phi(\mathbf{0}) \wedge \forall x(\phi(x) \rightarrow \phi(\mathbf{S} x))) \rightarrow \forall x \phi(x)$ where $\phi$ is a formula with at least one free variable $x$.

Now we first discuss some prominent fragments of $\mathbf{P A}$ extending $\mathbf{Q}$ in the literature.

We define the arithmetic hierarchy $I \Sigma_{n}$ and $B \Sigma_{n}$ in the language of PA. An $L$ (PA)-formula is bounded (or $\Delta_{0}$ formula) if all quantifiers occuring in it are bounded, i.e. in the form $(\exists x \leq y) \phi$ and $(\forall x \leq y) \phi$. For the definitions of $\Sigma_{n}, \Pi_{n}$ and $\Delta_{n}$ formulas $(n \geq 1)$, we refer to [25]. Collection for $\Sigma_{n+1}^{0}$ formulas is the following principle:

$$
(\forall x<u)(\exists y) \varphi(x, y) \rightarrow(\exists v)(\forall x<u)(\exists y<v) \varphi(x, y),
$$

where $\varphi(x, y)$ is a $\Sigma_{n+1}^{0}$ formula possibly containing parameters distinct from $u, v$.

The theory $I \Sigma_{n}$ is $\mathbf{Q}$ plus induction for $\Sigma_{n}$ formulas and $B \Sigma_{n+1}$ is $I \Sigma_{0}$ plus collection for $\Sigma_{n+1}$ formulas. It is well known that the following theories form a strictly increasing hierarchy:

$$
I \Sigma_{0}, B \Sigma_{1}, I \Sigma_{1}, B \Sigma_{2}, \cdots I \Sigma_{n}, B \Sigma_{n+1}, \cdots, \mathbf{P A} .
$$

Define $\omega_{1}(x)=x^{|x|}$ and $\omega_{n+1}(x)=2^{\omega_{n}(|x|)}$ where $|x|$ is the length of the binary expression of $x$. Note that the graphs of these functions can be defined in our language with the recursive defining equation provable (see [18]). Let $\Omega_{n}$ denote the statement $\forall x \exists y\left(\omega_{n}(x)=y\right)$ which says that $\omega_{n}(x)$ is total. There is a bounded formula $\operatorname{Exp}(\mathrm{x}, \mathrm{y}, \mathrm{z})$ such that $I \Sigma_{0}$ proves that $\operatorname{Exp}(\mathrm{x}, 0, \mathrm{z}) \leftrightarrow z=1$ and $\operatorname{Exp}(\mathrm{x}, \mathrm{Sy}, \mathrm{z}) \leftrightarrow \exists t(\operatorname{Exp}(\mathrm{x}, \mathrm{y}, \mathrm{t}) \wedge z=t \cdot x)$ (see [10, Proposition 2, p.299]). However, $I \Sigma_{0}$ cannot prove the totality of $\operatorname{Exp}(x, y, z)$. Let exp denote the statement postulating the totality of the exponential function $\forall x \forall y \exists z \operatorname{Exp}(\mathrm{x}, \mathrm{y}, \mathrm{z})$.

Theorem 2.3 ([16], [10]).

(1) For any $n \geq 1, I \Sigma_{0}+\Omega_{n}$ is interpretable in $\mathbf{Q}$ (see [10, Theorem 3, p.304]).

(2) $I \Sigma_{0}+\exp$ is not interpretable in $\mathbf{Q} \cdot 2$

(3) $I \Sigma_{1}$ is not interpretable in $I \Sigma_{0}+\exp$ (see [16, Theorem 1.1], p.186).

\footnotetext{
${ }^{2}$ See [10, Theorem 6, p.313]. Solovay proved that $I \Sigma_{0}+\neg \exp$ is interpretable in $\mathbf{Q}$ (see [10, Theorem 7, p.314]).
} 
(4) $I \Sigma_{n+1}$ is not interpretable in $B \Sigma_{n+1}$ (see [16, Theorem 1.2], p.186).

(5) $B \Sigma_{1}+\exp$ is interpretable in $I \Sigma_{0}+\exp$ (see [16, Theorem 2.4], p.188).

(6) For each $n \geq 1, B \Sigma_{1}+\Omega_{n}$ is interpretable in $I \Sigma_{0}+\Omega_{n}$ (see [16, Theorem 2.5], p.189).

(7) For each $n \geq 0, B \Sigma_{n+1}$ is interpretable in $I \Sigma_{n}$ (see [16, Theorem 2.6], p.189).

As a corollary, we have:

(1) The theories $\mathbf{Q}, I \Sigma_{0}, I \Sigma_{0}+\Omega_{1}, \cdots, I \Sigma_{0}+\Omega_{n}, \cdots, B \Sigma_{1}, B \Sigma_{1}+\Omega_{1}, \cdots, B \Sigma_{1}+$ $\Omega_{n}, \cdots$ are all mutually interpretable;

(2) $I \Sigma_{0}+\exp$ and $B \Sigma_{1}+\exp$ are mutually interpretable;

(3) For $n \geq 1, I \Sigma_{n}$ and $B \Sigma_{n+1}$ are mutually interpretable;

(4) $\mathbf{Q} \triangleleft I \Sigma_{0}+\mathbf{e x p} \triangleleft I \Sigma_{1} \triangleleft I \Sigma_{2} \triangleleft \cdots \triangleleft I \Sigma_{n} \triangleleft \cdots \triangleleft \mathbf{P A}$.

Now we discuss some weak theories in the literature which are mutually interpretable with $\mathbf{Q}$.

It is interesting to compare $\mathbf{Q}$ with its bigger brother $\mathbf{P A}^{-}$. The theory $\mathbf{P A}^{-}$is the theory of commutative, discretely ordered semi-rings with a minimal element plus the subtraction axiom. The theory $\mathbf{P A}^{-}$has the following axioms with $L\left(\mathbf{P A}^{-}\right)=L(\mathbf{P A}) \cup\{\leq\}:(1) x+0=x ;(2) x+y=$ $y+x ;(3)(x+y)+z=x+(y+z) ;(4) x \cdot 1=x ;$ (5) $x \cdot y=y \cdot x$; $(6)(x \cdot y) \cdot z=x \cdot(y \cdot z) ;(7) x \cdot(y+z)=x \cdot y+x \cdot z ;(8) x \leq y \vee y \leq x$; (9) $(x \leq y \wedge y \leq z) \rightarrow x \leq z ;(10) x+1 \not \leq x ;(11) x \leq y \rightarrow(x=y \vee x+1 \leq y)$; (12) $x \leq y \rightarrow x+z \leq y+z$; (13) $x \leq y \rightarrow x \cdot z \leq y \cdot z$; (14) $x \leq y \rightarrow$ $\exists z(x+z=y)$. From [37, $\mathbf{P A}{ }^{-}$is interpretable in $\mathbf{Q}$, and hence $\mathbf{P A}^{-}$is mutually interpretable with $\mathbf{Q}$.

Let $\mathbf{Q}^{+}$be the extension of $\mathbf{Q}$ with the following extra axioms $\left(L\left(\mathbf{Q}^{+}\right)=\right.$ $L(\mathbf{Q}) \cup\{\leq\})$ :

$$
\begin{aligned}
& \mathbf{Q}_{8}:(x+y)+z=x+(y+z) ; \\
& \mathbf{Q}_{9}: x \cdot(y+z)=x \cdot y+x \cdot z ; \\
& \mathbf{Q}_{10}:(x \cdot y) \cdot z=x \cdot(y \cdot z) ; \\
& \mathbf{Q}_{11}: x+y=y+x ; \\
& \mathbf{Q}_{12}: x \cdot y=y \cdot x ; \\
& \mathbf{Q}_{13}: x \leq y \leftrightarrow \exists z(x+z=y) .
\end{aligned}
$$

The theory $\mathbf{Q}^{+}$is interpretable in $\mathbf{Q}$ (see [10, Theorem 1], p.296), and hence $\mathbf{Q}^{+}$is mutually interpretable with $\mathbf{Q}$.

Andrzej Grzegorczyk considered a theory $\mathbf{Q}^{-}$in which addition and multiplication do satisfy natural reformulations of axioms of $\mathbf{Q}$ but are possibly non-total functions. More exactly, the language of $\mathbf{Q}^{-}$is $\{\mathbf{0}, \mathbf{S}, A, M\}$ where $A$ and $M$ are ternary relations, and the axioms of $\mathbf{Q}^{-}$are the axioms $\mathbf{Q}_{1}-\mathbf{Q}_{3}$ of $\mathbf{Q}$ plus the following six axioms about $A$ and $M$ :
A: $\forall x \forall y \forall z_{1} \forall z_{2}\left(A\left(x, y, z_{1}\right) \wedge A\left(x, y, z_{2}\right) \rightarrow z_{1}=z_{2}\right)$;
M: $\forall x \forall y \forall z_{1} \forall z_{2}\left(M\left(x, y, z_{1}\right) \wedge M\left(x, y, z_{2}\right) \rightarrow z_{1}=z_{2}\right)$;
G4: $\forall x A(x, \mathbf{0}, x)$;
G5: $\forall x \forall y \forall z(\exists u(A(x, y, u) \wedge z=\mathbf{S}(u)) \rightarrow A(x, \mathbf{S}(y), z))$;
G6: $\forall x M(x, \mathbf{0}, \mathbf{0})$;
G7: $\forall x \forall y \forall z(\exists u(M(x, y, u) \wedge A(u, x, z)) \rightarrow M(x, \mathbf{S}(y), z))$.

Andrzej Grzegorczyk asked whether $\mathbf{Q}^{-}$is essentially undecidable. Petr Hájek considered a somewhat stronger theory with axioms 
H5: $\forall x \forall y \forall z(\exists u(A(x, y, u) \wedge z=\mathbf{S}(u)) \leftrightarrow A(x, \mathbf{S}(y), z))$ and

H7: $\forall x \forall y \forall z(\exists u(M(x, y, u) \wedge A(u, x, z)) \leftrightarrow M(x, \mathbf{S}(y), z))$

instead of $\mathbf{G 5}$ and $\mathbf{G} 7$. He showed that this stronger variant of $\mathbf{Q}^{-}$is essentially undecidable (see [17]). Vítězslav Švejdar provided a positive answer to Grzegorczyk's original question in $[33$ and proved that $\mathbf{Q}$ is interpretable in $\mathbf{Q}^{-}$using the Solovay's method of shortening cuts (and hence $\mathbf{Q}^{-}$is essentially undecidable). Thus, $\mathbf{Q}^{-}$is mutually interpretable with $\mathbf{Q}$.

Andrzej Grzegorczyk proposed the theory of concatenation (TC) in [13] as a possible alternative theory for studying incompleteness and undecidability. Unlike Robinson (or Peano) Arithmetic, where the individuals are numbers that can be added or multiplied, in $\mathbf{T C}$ one has strings (or texts) that can be concatenated. We refer to [13] for Grzegorczyk's philosophical motivations to study TC.

The theory $\mathbf{T C}$ has the language $\{\frown, \alpha, \beta,=\}$ with a binary function symbol and two constants, and the following axioms:

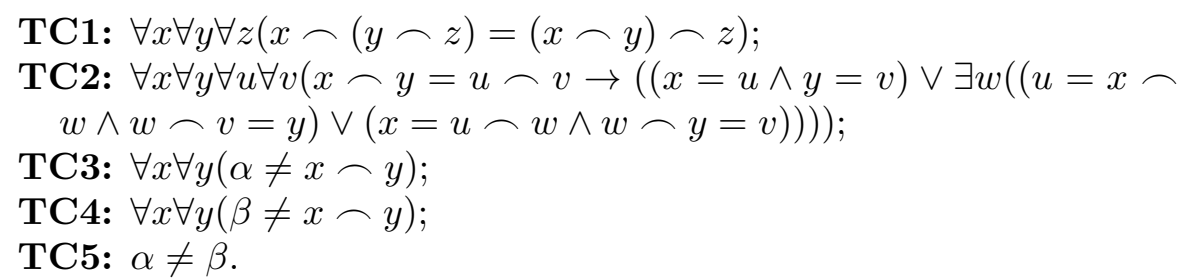

Grzegorczyk [13] proved (mere) undecidability of the theory TC. Grzegorczyk and Zdanowski [14] proved that TC is essentially undecidable. However, [14 leaves an interesting unanswered question: are TC and $\mathbf{Q}$ mutually interpretable? Švejdar 34] showed that $\mathbf{Q}^{-}$is interpretable in $\mathbf{T C}$ and hence $\mathbf{Q}$ is interpretable in $\mathbf{T C}$ since $\mathbf{Q}$ is interpretable in $\mathbf{Q}^{-}$. Ganea [11] gave a different proof of the interpretability of $\mathbf{Q}$ in $\mathbf{T C}$, but he also used the detour via $\mathbf{Q}^{-}$. Visser [38] gave a proof of the interpretability of $\mathbf{Q}$ in $\mathbf{T C}$ not using $\mathbf{Q}^{-}$. Note that $\mathbf{T C}$ is easily interpretable in the bounded arithmetic $I \Sigma_{0}$. Thus, $\mathbf{T C}$ is mutually interpretable with $\mathbf{Q}$.

Adjunctive Set Theory (AS) is the following theory in the language with only one binary relation symbol $\in$.

AS1: $\exists x \forall y(y \notin x)$.

AS2: $\forall x \forall y \exists z \forall u(u \in z \leftrightarrow(u=x \vee u=y))$.

The theory AS interprets Robinson's Arithmetic $\mathbf{Q}$, and hence is essentially undecidable. Nelson [26] showed that AS is interpretable in $\mathbf{Q}$. Thus, $\mathbf{A S}$ is mutually interpretable with $\mathbf{Q}$.

The theory $\mathbf{S}_{2}^{1}$ is a finitely axiomatizable weak arithmetic introduced by Buss in [3] to study polynomial time computability. The theory $\mathbf{S}_{2}^{1}$ gives us what we need to formalize the proof of the Second Incompleteness Theorem in a natural and effortless way. In fact, it is easier to do it in $\mathbf{S}_{\mathbf{2}}^{\mathbf{1}}$ than in $\mathbf{P A}$, since the restrictions present in $\mathbf{S}_{\mathbf{2}}^{1}$ prevent one from making wrong turns and inefficient choices (see [37]). From [10], $I \Sigma_{0}$ is interpretable in $\mathbf{S}_{2}^{1}$ and $\mathbf{S}_{2}^{\mathbf{1}}$ is interpretable in $\mathbf{Q}$. Thus, $\mathbf{S}_{\mathbf{2}}^{\mathbf{1}}$ is mutually interpretable with $\mathbf{Q}$.

Now, we introduce Robinson's theory $\mathbf{R}$ introduced by Tarski, Mostowski and Robinson in [35], and some variants of $\mathbf{R}$. 
Definition 2.4. Let $\mathbf{R}$ be the theory consisting of schemes $A \times 1-A \times 5$ with $L(\mathbf{R})=\{\mathbf{0}, \mathbf{S},+, \cdot, \leq\}$ where $\leq$ is a primitive binary relation symbol and $\bar{n}=\mathbf{S}^{n} \mathbf{0}$ for $n \in \mathbb{N}$ :

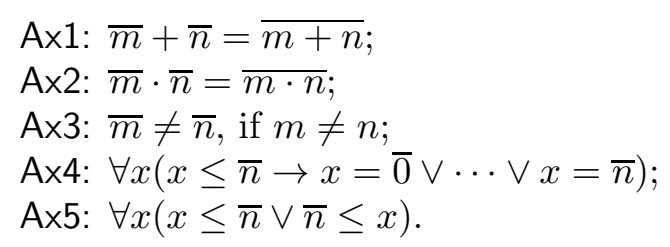

The axiom schemes of $\mathbf{R}$ contain all key properties of arithmetic for the proof of G1. The theory $\mathbf{R}$ is not finitely axiomatizable. Note that $\mathbf{R} \triangleleft \mathbf{Q}$ since $\mathbf{Q}$ is not interpretable in $\mathbf{R}$ : if $\mathbf{Q}$ is interpretable in $\mathbf{R}$, then it is interpretable in some finite fragment of $\mathbf{R}$; however $\mathbf{R}$ is locally finitely satisfiable but any model of $\mathbf{Q}$ is infinite. Visser [40] proved the following universal property of $\mathbf{R}$ which provides a unique characterization of $\mathbf{R}$.

Theorem 2.5 (Visser, Theorem 6, 40]). For any r.e. theory T, $T$ is locally finitely satisfiable iff $T$ is interpretable in $\mathbf{R} 3$

We say a specific class $\Phi$ of sentences has the finite model property if every satisfiable sentence of $\Phi$ has a finite model. Since relational $\Sigma_{2}$ sentences in a finite relational language have the finite model property (see Chapter 5 in [7]), by Theorem 2.5, any consistent theory axiomatized by a recursive set of $\Sigma_{2}$ sentences in a finite relational language is interpretable in $\mathbf{R}$. Since all recursive functions are representable in $\mathbf{R}$ (see [35, Theorem 6], p.56), from Theorem $2.1(2), \mathbf{R}$ is essentially undecidable. Cobham showed that $\mathbf{R}$ has a stronger property than essentially undecidability.

Theorem 2.6 (Cobham, [36]). Any r.e. theory that weakly interprets $\mathbf{R}$ is undecidable 4

Now, we discuss some variants of $\mathbf{R}$. If not explicitly mentioned otherwise, we assume that the base language is the same as $L(\mathbf{R})=\{\mathbf{0}, \mathbf{S},+, \cdot, \leq\}$ with $\leq$ as a primitive binary relation symbol. Let $\mathbf{R}_{0}$ be the theory consisting of schemes $A \times 1, A \times 2, A \times 3$ and $A \times 4$. The theory $\mathbf{R}_{0}$ is no longer essentially undecidable: the theory $\mathbf{R}_{0}$ has a decidable complete extension given by the theory of real closed fields with $\leq$ as the empty relation on reals. In fact, whether $\mathbf{R}_{0}$ is essentially undecidable depends on the language of $\mathbf{R}_{0}$. If $L\left(\mathbf{R}_{0}\right)=\{\mathbf{0}, \mathbf{S},+, \cdot\}$ with $\leq$ defined in terms of + , then $\mathbf{R}_{0}$ is essentially undecidable: Cobham first observed that $\mathbf{R}$ is interpretable in $\mathbf{R}_{0}$ and hence $\mathbf{R}_{0}$ is essentially undecidable (see [36] and [22]). Let $\mathbf{R}_{1}$ be the theory consisting of schemes $A \times 1, A \times 2, A \times 3$ and $A \times 4^{\prime}$ where $A \times 4^{\prime}$ is defined as follows:

$$
\forall x(x \leq \bar{n} \leftrightarrow x=\overline{0} \vee \cdots \vee x=\bar{n}) .
$$

The theory $\mathbf{R}_{1}$ is essentially undecidable since $\mathbf{R}$ is interpretable in $\mathbf{R}_{1}$ (see [22], p. 62). However $\mathbf{R}_{1}$ is not minimal essentially undecidable. Let $\mathbf{R}_{2}$ be the system consisting of schemes $A \times 2, A \times 3$ and $A \times 4^{\prime}$. From [22], $\mathbf{R}$ is

\footnotetext{
${ }^{3}$ In fact, if $T$ is locally finitely satisfiable, then $T$ is interpretable in $\mathbf{R}$ via a one-piece one-dimensional parameter-free interpretation.

${ }^{4}$ Vaught [36] gave a proof of Cobham's theorem via existential interpretation.
} 
interpretable in $\mathbf{R}_{2}$ and hence $\mathbf{R}_{2}$ is essentially undecidable 5 The theory $\mathbf{R}_{2}$ is minimal essentially undecidable in the sense that if we delete any axiom scheme of $\mathbf{R}_{2}$, then the remaining theory is not essentially undecidable: if we delete $\mathrm{A} \times 2$, then the theory of natural numbers with $x \cdot y$ defined as $x+y$ is a complete decidable extension; if we delete $A \times 3$, then the theory of models with only one element is a complete decidable extension; if we delete $A \times 4^{\prime}$, then the theory of real closed fields is a complete decidable extension. By essentially the same argument as the proof of Theorem 2.6 in [37], we can show that any r.e. theory that weakly interprets $\mathbf{R}_{2}$ is undecidable.

Kojiro Higuchi and Yoshihiro Horihata introduced the theory of concatenation $\mathbf{W} \mathbf{T C} \mathbf{C}^{-\epsilon}$, which is a weak subtheory of Grzegorczyk's theory $\mathbf{T C}$, and showed that $\mathbf{W} \mathbf{T} \mathbf{C}^{-\epsilon}$ is minimal essentially undecidable and $\mathbf{W} \mathbf{T} \mathbf{C}^{-\epsilon}$ is mutually interpretable with $\mathbf{R}$ (see [19]).

Elementary Arithmetic (EA) is $I \Delta_{0}+$ exp. We refer to 18 for the definition of Primitive Recursive Arithmetic (PRA). In a summary, we have the following pictures.

(1) Theories $\mathbf{P} \mathbf{A}^{-}, \mathbf{Q}^{+}, \mathbf{Q}, \mathbf{Q}^{-}, \mathbf{T C}, \mathbf{A} \mathbf{S}$ and $\mathbf{S}_{\mathbf{2}}^{\mathbf{1}}$ are mutually interpretable and are all essentially undecidable.

(2) Theories $\mathbf{R}, \mathbf{R}_{1}, \mathbf{R}_{2}$ and $\mathbf{W} \mathbf{T} \mathbf{C}^{-\epsilon}$ are mutually interpretable and are all essentially undecidable.

(3) $\mathbf{R} \triangleleft \mathbf{Q} \triangleleft \mathbf{E A} \triangleleft \mathbf{P R A} \triangleleft \mathbf{P A}$.

Now, a natural question is: among finitely axiomatized theories for which G1 holds, does $\mathbf{Q}$ have the least degree of interpretation? The following theorem tells us that the answer is no.

Theorem 2.7 (Visser, Theorem 2, [37]). Suppose $\mathbf{R} \subseteq A$, where A is finitely axiomatized and consistent. Then, there is a finitely axiomatized $B$ such that $\mathbf{R} \subseteq B \subseteq A$ and $B \triangleleft A$.

Theorem 2.7 shows that the structure $\langle\{S: \mathbf{R} \unlhd S \triangleleft \mathbf{Q}\}, \triangleleft\rangle$ is not well founded w.r.t. finitely axiomatized theories.

Theorem 2.8 (Visser, Theorem 12, 37]). Suppose $A$ and $B$ are finitely axiomatized theories that interpret $\mathbf{S}_{\mathbf{2}}^{\mathbf{1}}$. Then there are finitely axiomatized theories $\bar{A} \supseteq A$ and $\bar{B} \supseteq B$ such that $\bar{A}$ and $\bar{B}$ are incomparable (i.e. $\bar{A} \nsupseteq \bar{B}$ and $\bar{B} \notin \bar{A})$.

Theorem 2.8 shows that there are many pairs of incomparable theories extending $\mathbf{Q}$.

\section{Finding the Limit of APPLICABILITy OF G1 W.R.T. INTERPRETATION}

In this section, we examine the limit of applicability of G1 w.r.t. interpretation and show that we can find many theories weaker than $\mathbf{R}$ w.r.t. interpretation for which G1 holds based on Jeřábek's work using some model theory. First of all, we give some equivalent characterizations of the notion "G1 holds for the theory T".

\footnotetext{
${ }^{5}$ Another way to show that $\mathbf{R}_{2}$ is essentially undecidable is to prove that all recursive functions are representable in $\mathbf{R}_{2}$.
} 
Proposition 3.1. Let $T$ be a recursively axiomatizable consistent theory. The following are equivalent:

(1) G1 holds for $T$.

(2) $T$ is essentially incomplete.

(3) $T$ is essentially undecidable.

Proof. (1) $\Rightarrow(2)$ is trivial.

$(2) \Leftrightarrow(3)$ : It is well known that every consistent recursively axiomatizable complete theory is decidable; and every incomplete decidable theory has a consistent, decidable complete extension in the same language (see Corollary 3.1.8 and Theorem 3.1.9 in 25, p.214-215). From these two facts, $T$ is essentially undecidable iff $T$ is essentially incomplete.

$(2) \Rightarrow(1)$ : Follows from Theorem 2.1 and $(2) \Leftrightarrow(3)$.

As a corollary of Section 2, we have:

(1) G1 holds for the following theories and they are mutually interpretable: $\mathbf{Q}, I \Sigma_{0}, I \Sigma_{0}+\Omega_{n}(n \geq 1), B \Sigma_{1}, B \Sigma_{1}+\Omega_{n}(n \geq 1), \mathbf{T C}, \mathbf{Q}^{-}, \mathbf{Q}^{+}, \mathbf{P A} \mathbf{A}^{-}, \mathbf{S}_{\mathbf{2}}^{\mathbf{1}}$.

(2) G1 holds for the following theories and they are mutually interpretable: $\mathbf{R}, \mathbf{R}_{1}, \mathbf{R}_{2}$ and $\mathbf{W T C} \mathbf{C}^{-\epsilon}$.

Up to now, we do not have any example of an essentially undecidable theory $S$ such that $S \triangleleft \mathbf{R}$ and G1 holds for $S$. We find that Jeřábek's work in [21] essentially provides such an example of theory $S$. The motivating question of 21] is: if a theory represents all partial recursive functions, does it interpret Robinson's theory R? Jeřábek 21 negatively answered this question and showed that there exists a theory $T$ in which all partial recursive functions are representable, yet $T$ does not interpret $\mathbf{R}$. Jeřábek's proof uses tools from model theory: investigating model-theoretic properties of the model completion of the empty theory in a language with function symbols (see [21]) 6 Now we introduce Jeřábek's theory Repprf. Let PRF denote the set of all partial recursive functions. The language $L(\mathbf{R e p}$ PRF $)$ consists of constant symbols $\bar{n}$ for each $n \in \mathbb{N}$ and function symbols $\bar{f}$ of appropriate arity for each partial recursive function $f$. The theory RepPRF is axiomatized by:

(1) $\bar{n} \neq \bar{m}$ for $n \neq m \in \mathbb{N}$;

(2) $\bar{f}\left(\overline{n_{0}}, \cdots, \overline{n_{k-1}}\right)=\bar{m}$ for each $k$-ary partial recursive function $f$ such that $f\left(n_{0}, \cdots, n_{k-1}\right)=m$ where $n_{0}, \cdots, n_{k-1}, m \in \mathbb{N}$.

Corollary 3.2. G1 holds for $\mathbf{R e p} \mathrm{PRF}_{\text {and }} \mathbf{R e p} \mathrm{PRF}_{\mathrm{P} F} \triangleleft \mathbf{R}$.

Proof. The theory RepPRF is essentially undecidable since all recursive functions are representable in it. Since RepPRF is locally finitely satisfiable, by Theorem 2.5. $\mathbf{R e p}_{\mathrm{PRF}} \unlhd \mathbf{R}$. Jeřábek 21 showed that $\mathbf{R}$ is not interpretable in $\mathbf{R e p}$ PRF. Thus, G1 holds for $\mathbf{R e p}_{\mathrm{PRF}}$ and $\boldsymbol{R e p}_{\mathrm{PRF}} \triangleleft \mathbf{R}$.

Jeřábek's [21 is not written in the spirit of answering Question 1.5 and the potential of the method in [21] is not yet fully explored. Now, we give more examples of a theory $S$ such that G1 holds for $S$ and $S \triangleleft \mathbf{R}$ based on Jeřábek's work in 21.

\footnotetext{
${ }^{6} \mathrm{By}$ the empty theory, we mean the theory with no extra-logical axioms.
} 
Definition 3.3. We say $\langle S, T\rangle$ is a recursively inseparable pair if $S$ and $T$ are disjoint r.e. subsets of $\mathbb{N}$, and there is no recursive set $X \subseteq \mathbb{N}$ such that $S \subseteq X$ and $X \cap T=\emptyset$.

Definition 3.4. Let $\langle A, B\rangle$ be a recursively inseparable pair. Consider the following r.e. theory $U_{\langle A, B\rangle}$ with $L\left(U_{\langle A, B\rangle}\right)=\{\mathbf{0}, \mathbf{S}, \mathbf{P}\}$ where $\mathbf{P}$ is a unary relation symbol and $\bar{n}=\mathbf{S}^{n} \mathbf{0}$ for $n \in \mathbb{N}$ :

(1) $\bar{m} \neq \bar{n}$ if $m \neq n$;

(2) $\mathbf{P}(\bar{n})$ if $n \in A$;

(3) $\neg \mathbf{P}(\bar{n})$ if $n \in B$.

In the following, let $\langle A, B\rangle$ be an arbitrary recursively inseparable pair.

Lemma 3.5. G1 holds for $U_{\langle A, B\rangle}$.

Proof. By Proposition 3.1, it suffices to show that $U_{\langle A, B\rangle}$ is essentially incomplete. let $S$ be a recursively axiomatizable consistent extension of $U_{\langle A, B\rangle}$. Let $X=\{n: S \vdash \mathbf{P}(\bar{n})\}$ and $Y=\{n: S \vdash \neg \mathbf{P}(\bar{n})\}$. Then $A \subseteq X$ and $B \subseteq Y$. Since $S$ is recursively axiomatizable and consistent, $X$ and $Y$ are disjoint recursive enumerable sets. Since $\langle A, B\rangle$ is recursively inseparable, $X \cup Y \neq \mathbb{N}$. Take $n \notin X \cup Y$. Then $S \nvdash \mathbf{P}(\bar{n})$ and $S \nvdash \neg \mathbf{P}(\bar{n})$. Hence $S$ is incomplete.

Fact 3.6 (Theorem 2, p.43, 24]). Let $A$ and $B$ be disjoint r.e. subsets of $\mathbb{N}$ and $T$ be a consistent r.e. extension of $\mathbf{Q}$. Then there is a $\Sigma_{1}$ formula $\phi(x)$ such that for any $n$, we have:

(1) $n \in A$ iff $T \vdash \phi(\bar{n})$;

(2) $n \in B$ iff $T \vdash \neg \phi(\bar{n})$.

Lemma 3.7. The theory $U_{\langle A, B\rangle}$ is interpretable in $\mathbf{R}$.

Proof. By Fact 3.6, there exists a formula $\phi(x)$ with one free variable such that $\mathbf{R} \vdash \phi(\bar{n})$ iff $n \in A$ and $\mathbf{R} \vdash \neg \phi(\bar{n})$ iff $n \in B \sqrt{ }$ Thus $U_{\langle A, B\rangle}$ is interpretable in $\mathbf{R}$ via interpreting $\mathbf{P}(x)$ as $\phi(x)$.

Here is another proof of Lemma 3.7; since $U_{\langle A, B\rangle}$ is a locally finitely satisfiable r.e. theory, by Theorem 2.5, $U_{\langle A, B\rangle}$ is interpretable in $\mathbf{R}$.

Based on Jeřábek's work in [21], our strategy to prove that $U_{\langle A, B\rangle}$ does not interpret $\mathbf{R}$ is to consistently extend the interpreting theory to a theory with quantifier elimination, using the fact that the empty theory in an arbitrary language $L$ has a model completion which we denote by $\mathbf{E C}_{L}$, the theory of existentially closed $L$-structures. The theory $\mathbf{E C}_{L}$ admits the elimination of quantifiers (see [21]). The two key tools we use to show that $U_{\langle A, B\rangle}$ does not interpret $\mathbf{R}$ are Theorem 3.8 and Theorem 3.9 which essentially use properties of $\mathbf{E C}_{L}$. We say a relation $R \subseteq X^{2}$ is asymmetric if there are no $a, b \in X$ such that $R(a, b)$ and $R(b, a)$.

Theorem 3.8 (Jeřábek, Theorem 5.1, [21]). For any first order language $L$ and formula $\phi(\bar{z}, \bar{x}, \bar{y})$ with $\operatorname{lh}(\bar{x})=\operatorname{lh}(\bar{y})$, there is a constant $n$ with the following property. Let $M \models \mathbf{E C}_{L}$ and $\bar{u} \in M$ be such that $M \models$

\footnotetext{
${ }^{7}$ The proof of Fact 3.6] in [24, Theorem 2, p.43] uses the fixed point theorem for the base theory $T$. Since the fixed point theorem holds for $\mathbf{R}$, Fact 3.6 also applies to $\mathbf{R}$.
} 
$\exists \bar{x}_{0}, \cdots, \exists \bar{x}_{n-1} \bigwedge_{i<j<n} \phi\left(\bar{u}, \bar{x}_{i}, \bar{x}_{j}\right)$. Then for every $m \in \mathbb{N}$ and an asymmetric relation $R$ on $\{0, \cdots, m-1\}, M \models \exists \bar{x}_{0}, \cdots, \exists \bar{x}_{m-1} \bigwedge_{\langle i, j\rangle \in R} \phi\left(\bar{u}, \bar{x}_{i}, \bar{x}_{j}\right)$.

Theorem 3.9 (Jeřábek, Theorem 4.5, [21]). For a $\Sigma_{2}$-axiomatized theory $T, T$ is interpretable in a consistent existential theory iff $T$ is weakly interpretable in $\mathbf{E C}_{L}$ for some language $L$.

Especially, Jeřábek 21] showed that: (1) if a theory is interpretable in a consistent quantifier-free or existential theory, it is weakly interpretable in $\mathbf{E C}_{L}$ for some language $L$, and the interpretation can be taken quantifierfree; (2) if a $\Sigma_{2}$ theory is weakly interpretable in $\mathbf{E C}_{L}$, it is interpretable in a quantifier-free theory.

Definition 3.10. Consider the following theory $\mathbf{T}$ in the language $\langle\epsilon\rangle$ axiomatized by the sentences $\exists z, x_{0}, \cdots, x_{n-1}\left(\bigwedge_{i<j<n} x_{i} \neq x_{j} \wedge \forall y(y \in z \leftrightarrow\right.$ $\left.\bigvee_{i<n} y=x_{i}\right)$ ) for all $n \in \mathbb{N}$.

\section{Proposition 3.11.}

(1) $\mathbf{T}$ is not weakly interpretable in $\mathbf{E} \mathbf{C}_{L}$ for any language $L$.

(2) $\mathbf{R}$ is not weakly interpretable in $\mathbf{E C}_{L}$ for any language $L$.

(3) If $\mathbf{R}$ is interpretable in $U_{\langle A, B\rangle}$, then $\mathbf{R}$ is weakly interpretable in $\mathbf{E C}_{L}$ for some language $L$.

Proof. (1): Suppose this does not hold and apply Theorem 3.8 to the formula which interprets $\bigwedge_{i<j<n} x_{i} \neq x_{j} \wedge \forall y\left(y \in z \leftrightarrow \bigvee_{i<n} y=x_{i}\right)$ and $R$ a chain longer than $n$ to get a contradiction.

(2): Note that $\mathbf{T}$ is interpretable in $\mathbf{R}$. Since $\mathbf{T}$ is not weakly interpretable in $\mathbf{E C}_{L}$ for any language $L, \mathbf{R}$ is not weakly interpretable in $\mathbf{E} \mathbf{C}_{L}$ for any language $L$.

(3): This follows from Theorem 3.9 since $U_{\langle A, B\rangle}$ is a consistent r.e. theory.

Theorem 3.12. For any recursively inseparable pair $\langle A, B\rangle$, there is a r.e. theory $U_{\langle A, B\rangle}$ such that $\mathrm{G} 1$ holds for $U_{\langle A, B\rangle}$ and $U_{\langle A, B\rangle} \triangleleft \mathbf{R} 8$

Proof. By Proposition $3.11(2)-(3), \mathbf{R}$ is not interpretable in $U_{\langle A, B\rangle}$. From Lemma 3.5 and Lemma 3.7, we have G1 holds for $U_{\langle A, B\rangle}$ and $U_{\langle A, B\rangle} \triangleleft \mathbf{R}$.

Corollary 3.13. Let $S$ be a consistent existential theory. Then the following are equivalent:

(1) G1 holds for $S$ and $S \triangleleft \mathbf{R}$ (i.e. $S$ is a solution for Question 1.5);

(2) $S$ is essentially undecidable and locally finitely satisfiable.

Proof. As a corollary of Theorem 3.9 and Proposition 3.11(2), any consistent existential theory does not interpret R. Thus, from this, Proposition 3.1 and Theorem 2.5, we have the equivalence.

From Theorem 2.5, $\mathbf{R}$ has the universality property: every locally finitely satisfiable r.e. theory is interpretable in it. Albert Visser asked the following question:

\footnotetext{
${ }^{8}$ However, Theorem 3.12 does not tell us more information about the theory $U_{\langle S, T\rangle}$ and $U_{\langle U, V\rangle}$ for different recursively inseparable pairs $\langle S, T\rangle$ and $\langle U, V\rangle$ : e.g. whether $U_{\langle S, T\rangle}$ and $U_{\langle U, V\rangle}$ have the same degree of interpretation or the same degree of Turing reducibility.
} 
Question 3.14 (Visser). Would $S$ with $S \unlhd \mathbf{R}$ such that G1 holds for $S$ share the universality property of $\mathbf{R}$ that every locally finitely satisfiable r.e. theory is interpretable in it.

As a corollary of Theorem 3.12, the answer for this question is negative. We have shown that for any recursively inseparable pair $\langle A, B\rangle$, there is a theory $U_{\langle A, B\rangle}$ such that $\mathrm{G} 1$ holds for $U_{\langle A, B\rangle}$ and $U_{\langle A, B\rangle} \triangleleft \mathbf{R}$. The theory $\mathbf{R}$ is locally finitely satisfiable, but $\mathbf{R}$ is not interpretable in $U_{\langle A, B\rangle}$. Take another example: the theory $\mathbf{T}$ as in Definition 3.10 is locally finitely satisfiable, but $\mathbf{T}$ is not interpretable in $U_{\langle A, B\rangle}$ (if $\mathbf{T}$ is interpretable in $U_{\langle A, B\rangle}$, by Theorem 3.9. $\mathbf{T}$ is weakly interpretable in $\mathbf{E C}_{L}$ for some language $L$ which contradicts Proposition $3.11(1))$. Thus, for any recursively inseparable pair $\langle A, B\rangle$, the theory $U_{\langle A, B\rangle}$ is a counterexample for Visser's Question. This shows the speciality of $\mathbf{R}$ : Theorem 2.5 provides a unique characterization of $\mathbf{R}$.

Define $\mathrm{D}=\{S: S \triangleleft \mathbf{R}$ and G1 holds for the theory $S\}$. We have shown that we could find many witnesses for $D$. We could naturally examine the structure of $\langle\mathrm{D}, \triangleleft\rangle$. A natural question is: whether the similar results as in Theorem 2.7 and Theorem 2.8 apply to the structure $\langle\mathrm{D}, \triangleleft\rangle$. About the structure of $\langle\mathrm{D}, \triangleleft\rangle$, we could naturally ask:

\section{Question 3.15.}

(1) Is $\langle\mathrm{D}, \triangleleft\rangle$ well founded (or is it that for any $S \in \mathrm{D}$, there is $T \in \mathrm{D}$ such that $T \triangleleft S)$ ?

(2) Are any two elements of $\langle\mathrm{D}, \triangleleft\rangle$ comparable (i.e. is it that for any $S, T \in$ D, we have either $S \unlhd T$ or $T \unlhd S)$ ?

(3) Could we find a theory $S$ with a minimal degree of interpretation such that G1 holds for $S$ ?

In the rest of this paper, we will show that if we consider the Turing degree structure instead of the interpretation degree structure of $D$, we have definite answers for Question 3.15.

\section{The limit of APplicability of G1 W.R.T. Turing Reducibility}

In this section, we examine the limit of applicability of G1 w.r.t. Turing reducibility and show that there is no theory with a minimal degree of Turing reducibility for which G1 holds based on Shoenfield's work using some recursion theory.

Let $\mathcal{R}$ be the structure of the r.e. degrees with the ordering $\leq_{T}$ induced by Turing reducibility with the least element $\mathbf{0}$ and the greatest element $\mathbf{0}^{\prime}$. Define $\overline{\mathrm{D}}=\left\{S: S<_{T} \mathbf{R}\right.$ and $\mathrm{G} 1$ holds for the theory $\left.S\right\}$. A natural question is to examine the structure of $\left\langle\overline{\mathrm{D}},<_{T}\right\rangle$. Now, we will show that the structure $\left\langle\overline{\mathrm{D}},<_{T}\right\rangle$ is much simpler than $\langle\mathrm{D}, \triangleleft\rangle$ and we have answers to Question 3.15 for the structure $\left\langle\overline{\mathrm{D}},<_{T}\right\rangle$ based on Shoenfield's work using some recursion theory.

Theorem 4.1 (Shoenfield, Theorem 1, 30]). If $A$ is recursively enumerable and not recursive, there is a recursively inseparable pair $\langle B, C\rangle$ such that $A$, $B$ and $C$ have the same Turing degree.

Now, we will show that for any Turing degree $\mathbf{0}<\mathbf{d}<\mathbf{0}^{\prime}$, there is a theory $U$ such that $\mathrm{G} 1$ holds for $U, U<_{T} \mathbf{R}$ and $U$ has Turing degree $\mathbf{d}$ 
(c.f. Theorem 4.5). The following theorem of Shoenfield is essential for the proof of Theorem 4.5. To make the reader have a better sense of how the theory $U$ in Theorem 4.5 is constructed, we provide details of the proof of Theorem 4.2. Feferman [9] also proved that for any r.e. Turing degree one can design a formal theory whose corresponding decision problem is of the same degree (however, it is not clear whether such a formal theory is essentially undecidable).

Theorem 4.2 (Shoenfield, Theorem 2, 30]). Let A be recursively enumerable and not recursive. Then there is a consistent axiomatizable theory $T$ having one non-logical symbol which is essentially undecidable and has the same Turing degree as $A$.

Proof. By Theorem 4.1, pick a recursively inseparable pair $\langle B, C\rangle$ such that $A, B$ and $C$ have the same Turing degree. Now we define the theory $T$ with $L(T)=\{R\}$ where $R$ is a binary relation symbol. Theory $T$ contains axioms asserting that $R$ is an equivalence relation. Let $\Phi_{n}$ be the statement that there is an equivalence class of $R$ consisting of $n$ elements. Then, as axioms of $T$ we adopt $\Phi_{n}$ for all $n \in B$ and $\neg \Phi_{n}$ for all $n \in C$. Finally, for each $n$ we adopt an axiom asserting there is at most one equivalence class of $R$ having $n$ elements. Clearly, $T$ is consistent and axiomatizable. Using models, we see $\Phi_{n}$ is provable iff $n \in B$, and $\neg \Phi_{n}$ is provable iff $n \in C$. Hence $B$ and $C$ are recursive in $T$.

Disjunctions of conjunctions whose terms are $\Phi_{n}$ or $\neg \Phi_{n}$ for some $n \in \mathbb{N}$, are called a disjunctive normal form of $\left\langle\Phi_{n}: n \in \mathbb{N}\right\rangle$.

Lemma 4.3 (Janiczak, Lemma 2 in [20]). Any sentence $\phi$ of the theory $T$ is equivalent to a disjunctive normal form of $\left\langle\Phi_{n}: n \in \mathbb{N}\right\rangle$, and this disjunctive normal form can be found explicitly once $\phi$ is explicitly given 9

By Lemma 4.3, every sentence $\phi$ of $T$ is equivalent to a disjunctive normal form of $\left\langle\Phi_{n}: n \in \mathbb{N}\right\rangle$, and this disjunctive normal form can be calculated from $\phi$. It follows that $T$ is recursive in $B$ and $C$. Hence $T$ has the same Turing degree as $A$.

Finally, we show that $T$ is essentially undecidable. Suppose $T$ has a consistent decidable extension $S$. Let $D$ be the set of $n$ such that $\Phi_{n}$ is provable in $S$. Then $D$ is recursive, $B \subseteq D$, and $C \cap D=\emptyset$ which contradicts the fact that $\langle B, C\rangle$ is a recursively inseparable pair.

Theorem 4.4 (Sacks).

(1) (Embedding theorem, 28] Every countable partial ordering can be embedded into $\mathcal{R}$.

(2) (Density Theorem, 29]) For every pair of nonrecursive r.e. degrees $\mathbf{a}<_{T}$ $\mathbf{b}$, there is one $\mathbf{c}$ such that $\mathbf{a}<_{T} \mathbf{c}<_{T} \mathbf{b}$.

Theorem 4.5. For any Turing degree $\mathbf{0}<\mathbf{d}<\mathbf{0}^{\prime}$, there is a theory $U$ such that $\mathrm{G} 1$ holds for $U, U<_{T} \mathbf{R}$ and $U$ has Turing degree $\mathbf{d}$.

Proof. From Theorem 4.2, for each Turing degree $\mathbf{0}<\mathbf{d}<\mathbf{0}^{\prime}$, there is a theory $U$ such that $\mathrm{G} 1$ holds for $U$ and $U$ has Turing degree $\mathbf{d}$. It is a well known fact that $\mathbf{R}$ has Turing degree $\mathbf{0}^{\prime}$.

\footnotetext{
${ }^{9}$ This is a reformulation of Janiczak's Lemma 2 in [20] in the context of the theory $T$. Janiczak's Lemma is proved by means of a method known as the elimination of quantifiers.
} 
We could ask the similar question as Question 3.15 for the structure $\left\langle\overline{\mathrm{D}},<_{T}\right\rangle$ :

- Is $\left\langle\overline{\mathrm{D}},<_{T}\right\rangle$ well founded?

- Are any two elements of $\left\langle\overline{\mathrm{D}},<_{T}\right\rangle$ comparable?

- Could we find a theory $S$ with a minimal degree of Turing reducibility such that G1 holds for $S$ ?

From Theorem 4.5 and Theorem 4.4 , we have answers for these questions:

\section{Corollary 4.6.}

(1) The structure $\left\langle\overline{\mathrm{D}},<_{T}\right\rangle$ is not well founded (i.e. for any $S \in \overline{\mathrm{D}}$, there is $U \in \overline{\mathrm{D}}$ such that $\left.U<_{T} S\right)$;

(2) The structure $\left\langle\overline{\mathrm{D}},<_{T}\right\rangle$ has incomparable elements (i.e. there are $U, V \in$ $\overline{\mathrm{D}}$ such that $U \mathbb{Z}_{T} V$ and $\left.V \mathbb{\leq}_{T} U\right)$;

(3) There is no theory with a minimal degree of Turing reducibility for which G1 holds.

In fact, we can improve Theorem 4.5 by making that the theory $U$ is interpretable in $\mathbf{R}$.

Theorem 4.7. For any Turing degree $\mathbf{0}<\mathbf{d}<\mathbf{0}^{\prime}$, there is a theory $U$ such that $\mathrm{G} 1$ holds for $U, U \unlhd \mathbf{R}$ and $U$ has Turing degree $\mathbf{d}$.

Proof. Let $\mathbf{d}$ be a Turing degree with $\mathbf{0}<\mathbf{d}<\mathbf{0}^{\prime}$. By Theorem 4.2, pick an essentially undecidable theory $S$ with Turing degree $\mathbf{d}$.

Consider the product theory $S \otimes \mathbf{R}$ defined as follows. The theory $S \otimes \mathbf{R}$ has the following axioms: $P \rightarrow X$ if $X$ is a $S$-axiom; $\neg P \rightarrow Y$ if $Y$ is a $\mathbf{R}$-axiom where $P$ is a 0 -ary predicate symbol.

Now, we show that $S \otimes \mathbf{R}$ is essentially undecidable (i.e. G1 holds for $S \otimes \mathbf{R})$ and interpretable in $\mathbf{R}$.

Lemma 4.8. $S \otimes \mathbf{R}$ is essentially undecidable.

Proof. Suppose $U$ is a consistent decidable extension of $S \otimes \mathbf{R}$. Define $X=\{\langle\ulcorner\phi\urcorner,\ulcorner\psi\urcorner\rangle: U \vdash P \rightarrow \phi$ or $U \vdash \neg P \rightarrow \psi\}$. Since $U$ is decidable, $X$ is recursive. Note that $S \subseteq(X)_{0}$ and $\mathbf{R} \subseteq(X)_{1}$. We claim that at least one of $(X)_{0}$ and $(X)_{1}$ is consistent. If both $(X)_{0}$ and $(X)_{1}$ are inconsistent, then $U \vdash(P \rightarrow \perp)$ and $U \vdash(\neg P \rightarrow \perp)$. Thus, $U \vdash \perp$ which contradicts that $U$ is consistent. WLOG, we assume that $(X)_{0}$ is consistent. Then $(X)_{0}$ is consistent decidable extension of $S$ which contradicts that $S$ is essentially undecidable.

It is easy to show that $S \otimes \mathbf{R}$ is interpretable in $\mathbf{R}$ (i.e. $S \otimes \mathbf{R} \unlhd \mathbf{R}$ ): take the identity interpretation on the $\mathbf{R}$ side and interpret $P$ as $\perp$.

Since $S$ has Turing degree $\mathbf{d}$ and $\mathbf{R}$ has Turing degree $\mathbf{0}^{\prime}, S \otimes \mathbf{R}$ has Turing degree $\mathbf{d}$.

However, from the proof of Theorem 4.7, we cannot get that $S \otimes \mathbf{R} \triangleleft \mathbf{R}$ (i.e. $\mathbf{R}$ is not interpretable in $S \otimes \mathbf{R}$ ). An interesting question is: could we improve Theorem 4.7 and show that for any Turing degree $\mathbf{0}<\mathbf{d}<\mathbf{0}^{\prime}$, there is a theory $U$ such that $\mathrm{G} 1$ holds for $U, U \triangleleft \mathbf{R}$ and $U$ has Turing degree $\mathbf{d}$.

As far as we know, Question 3.15 is open. We make the conjecture that there is no theory with a minimal degree of interpretation for which G1 holds, $\langle\mathrm{D}, \triangleleft\rangle$ is not well founded and $\langle\mathrm{D}, \triangleleft\rangle$ has incomparable elements. 


\section{REFERENCES}

[1] Lev D. Beklemishev. Gödel incompleteness theorems and the limits of their applicability I. Russian Math Surveys, 2010.

[2] George Boolos. The Logic of Provability. Cambridge University Press, 1993.

[3] Samuel R. Buss. Bounded Arithmetic. Bibliopolis, Napoli, 1986.

[4] Yong Cheng. Incompleteness for Higher-Order Arithmetic: An Example Based on Harrington's Principle. Springer series: Springerbrief in Mathematics, Springer, 2019.

[5] Yong Cheng. Current research on Gödel's incompleteness theorems. Submitted, DOI: 10.13140/RG.2.2.28820.65924, 2019.

[6] Yong Cheng. On the depth of Gödel's incompleteness theorem. Submitted, DOI: 10.13140/RG.2.2.20658.56004, 2020.

[7] Heinz-Dieter Ebbinghaus and Jorg Flum. Finite Model Theory. Springer Monographs in Mathematics, Springer, 1999.

[8] Herbert B. Enderton. A mathematical introduction to logic (2nd ed.). Boston, MA: Academic Press, 2001.

[9] Solomon Feferman. Degrees of unsolvability associated with classes of formalized theories. Journal of Symbolic Logic, 22(2):161-175, 1957.

[10] Fernando Ferreira and Gilad Ferreira. Interpretability in Robinson's Q. The Bulletin of Symbolic Logic, Vol. 19, No. 3 (September 2013), pp. 289-317.

[11] Mihai Ganea. Arithmetic on semigroups. J. Symb. Logic, 74(1):265-278, 2009.

[12] Kurt Gödel. Über formal unentscheidbare Sätze der Principia Mathematica und verwandter Systeme I. Monatsh. Math. Phys. 38:1 (1931), 173-198.

[13] Andrzej Grzegorczyk. Undecidability without arithmetization. Studia Logica, 79(2):163-230, 2005.

[14] Andrzej Grzegorczyk and Konrad Zdanowski. Undecidability and concatenation. In A. Ehrenfeucht, V. W. Marek, M. Srebrny (Eds.), Andrzej Mostowski and foundational studies (pp. 72-91). Amsterdam: IOS Press, 2008.

[15] David Guaspari and Robert M. Solovay. Rosser sentences. Annals of Mathematical Logic, 16(1), 81-99, 1979.

[16] Petr Hájek. Interpretability and Fragments of Arithmetic. Arithmetic, Proof Theory and Computational Complexity (Peter Clote; Jan Krajícek, J.). Oxford Logic Guides, 23, p. 185-196. Oxford: Clarendon Press, 1993.

[17] Petr Hájek. Mathematical fuzzy logic and natural numbers. Fundamenta Informaticae, 81 (1-3), 155-163, 2007.

[18] Petr Hájek and Pavel Pudlák. Metamathematics of First-Order Arithmetic. SpringerVerlag, Berlin-Heidelberg-New York, 1993.

[19] Kojiro Higuchi and Yoshihiro Horihata. Weak theories of concatenation and minimal essentially undecidable theories-an encounter of WTC and S2S. Arch. Math. Log., 53(7-8):835-853, 2014.

[20] Antoni Janiczak. Undecidability of some simple formalized theories. Fundamenta Mathematicae, vol. 40 (1953), pp. 131-139.

[21] Emil Jeřábek. Recursive functions and existentially closed structures. To appear in Journal of Mathematical Logic.

[22] James P. Jones and John C. Shepherdson. Variants of Robinson's essentially undecidable theory R. Archive Math. Logic, 23, 65-77, 1983.

[23] Henryk Kotlarski. The incompleteness theorems after 70 years. Annals of Pure and Applied Logic 126, 125-138, 2004.

[24] Per Lindström. Aspects of Incompleteness. Lecture Notes in Logic v. 10, 1997.

[25] Roman Murawski. Recursive Functions and Metamathematics: Problems of Completeness and Decidability, Gödel's Theorems. Springer Netherlands, 1999.

[26] Edward Nelson. Predicative arithmetic. Mathematical Notes, Princeton University Press, 1986.

[27] Marian Boykan Pour-El and Saul Kripke. Deduction-preserving "recursive isomorphisms" between theories. Fundam Math 61:141-163, 1967. 
[28] Gerald E. Sacks. Degrees of unsolvability. Annals of Math. Studies 55, Princeton Univ. Press, Princeton NJ, 1963.

[29] Gerald E. Sacks. The recursively enumerable degrees are dense. Ann. of Math. (2) 80, 300-312, 1964.

[30] Joseph R. Shoenfield. Degrees of formal systems. The Journal of Symbolic Logic, Volume 23, Number 4, Dec. 1958.

[31] Peter Smith. An Introduction to Gödel's Theorems. Cambridge University Press, 2007.

[32] Craig Smoryński. The Incompleteness Theorems. in: J. Barwise (Ed.), Handbook of Mathematical Logic, North-Holland, Amsterdam, 1977, pp. 821-865.

[33] Vítězslav Švejdar. An interpretation of Robinson arithmetic in its Grzegorczyk's weaker variant. Fundamenta Informaticae, 81(1-3):347-354, 2007.

[34] Vítězslav Švejdar. On Interpretability in the Theory of Concatenation. Notre Dame Journal of Formal Logic Volume 50, Number 1, 2009.

[35] Alfred Tarski, Andrzej Mostowski and Raphael M. Robinson. Undecidabe theories. Studies in Logic and the Foundations of Mathematics, North-Holland, Amsterdam, 1953.

[36] Robert L. Vaught. On a theorem of Cobham concerning undecidable theories. In: Nagel E, Suppes P, Tarski A (eds), Proceedings of the 1960 international congress on logic, methodology and philosophy of science. Stanford University Press, Stanford, pp 14-25, 1962.

[37] Albert Visser. On Q. Soft Comput. DOI 10.1007/s00500-016-2341-5, 2016.

[38] Albert Visser. Growing commas: a study of sequentiality and concatenation. Notre Dame J. Formal Logic, 50(1):61-85, 2009.

[39] Albert Visser. Can we make the second incompleteness theorem coordinate free? Journal of Logic and Computation 21(4), 543-560, 2011.

[40] Albert Visser. Why the theory $\mathbf{R}$ is special? In Foundational Adventures. Essay in honour of Harvey Friedman, pages 7-23. College Publications, 2014.

[41] Albert Visser. The Second Incompleteness Theorem: Reflections and Ruminations. Chapter in Gödel's Disjunction: The scope and limits of mathematical knowledge, edited by Leon Horsten and Philip Welch, Oxford University Press, 2016.

School of Philosophy, Wuhan University, Wuhan 430072 Hubei, Peoples Republic of China

E-mail address: world-cyr@hotmail.com 